\title{
Flow Assisted Flame Spread over Thermally Thin Fuels
}

\author{
H. T. LOH and A. C. FERNANDEZ-PELLOO \\ Department of Mechanical Engineering \\ University of California \\ Berkeley, California 94720 , USA
}

\section{Abstract}

Small scale experiments have been conducted of the spread of flames over the surface of thin filter paper sheets in a mixed convective flow moving in the direction of flame spread. The rate of flame spread has been measured as a function of the flow velocity and oxygen concentration. It is found that after an initial accelerating stage, the flame spreadrate becomes constant as the flame progresses over the fuel surface. The initial acceleration period becomes shorter as the oxygen concentration is increased. The spread rate is weakly dependent on the flow velocity for low gas velocities (mixed flow) but becomes independent of the flow velocity for forced flow conditions. The flame spread rate is practically linearly dependent on the oxygen concentration of the flow over the range of concentrations tested. The data for the rate of flame spread can be correlated in terms of an expression of the spread rate that is obtained from a simplified heat transfer model of the flame spread process.

\section{Introduction}

Flame spread over solid combustibles is an important process during the growth of a fire. Although a considerable amount of research has been performed over the last few years on the flow assisted mode of flame spread, the complexity of the problem has so far prevented a complete understanding of the processes.

The concurrent mode of flame spread is characterized by a flame spreading over the surface of a solid combustible in a gaseous oxidizer

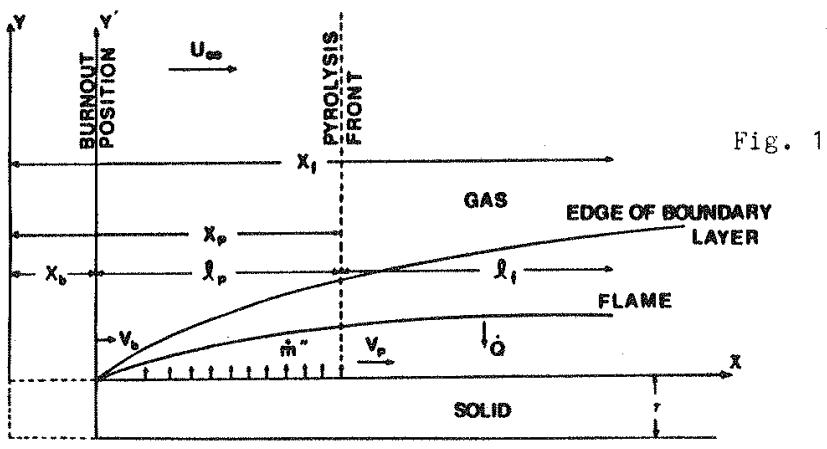

Schematic diagram of model of flow assisted flame spread and coordinate system. 
that flows in the same direction as that of flame propagation (Fi8. 1 ). The fuel vapor that is not completely consumed by the upstream flame is induced by convection downstream ahead of the pyrolysis front. This fuel vapor ifeps reacting with the oxidizer and thus extends the diffusion flame downstream from the pyrolysis front. The proximity of the flame and the post-combustion gases to the flel surface favors the heat transfer to the unburnt combustible. As a consequence, the resulting flame spread process is generally rapid and hazardous.

Most experimental studies of the flow assisted mode of flame spread have been performed with thick fuels [1-6]. The only studies performed with thin fuels are the experiments of Markstein and de Ris [7] with cotton sheets, and Hirano and Sato [8] and Hirano et al [9] with paper sheets. In all cases the flames spread in an air flow. The reader is referred to the recent review of ref. [10] for an overview of these works. To the best knowledge of the authors, no studies have been performed on the spread of flames over thin fuel in a flow of varied oxygen concentration. Although the processes of flame spread over the surface of thick or thin combustible materials are basically the same, there are two major characteristics that differenciate one from another. One is that the fuel is consumed in the upstream region of the fuel sheet producing a propagating "burn out" front in addition to the propagating pyrolysis and flame sheet fronts common in both thin and thick fuels. Another difference is that in a thermally thin fuel the temperature is uniform accross its thickness, while in a thermally thick fuel its thickness does not affect the temperature distribution. These two basic differences may result in different controlling mechanisms of the flame spread processes for thin and thick combustibles.

In the present work an experimental study is carried out on the spread of flames over the surface of thin filter paper sheets in a forced flow with varied velocity and oxygen concentration moving in the direction of flame spread. The objective of the work is to provide basic information of the controlling mechanisms of flame spread. of particular importance is the study of the influence of the burn-out process of the fuel on the flame spread rate.

\section{Experiment}

A schematic diagram of the experimental installation is shown in Fig. 2. The experiments are carried out in a small scale combustion tunnel with a test section that has a rectangular cross section of $0.127 \mathrm{~m}$ wice, 0.0762 $\mathrm{m}$ deep, and is $0.61 \mathrm{~m}$ long [6]. The walls of the test section are made of pyrex glass to allow optical access to the test area. The fuel specimens are $0.33 \mathrm{~mm}$ thick whatman chromatography paper, $0.076 \mathrm{~m}$ wide by $0.45 \mathrm{~m}$ long. The paper sheets are mounted in a metalile frame by inserting them in metallic spikes placed on the sides of the frame. The spikes are used to hold paper in slight tension to provide a $\mathrm{f}^{2}$ lat surface [8]. In order to generate a flat plate flow over both surfaces of the fuel sheet, the paper is positioned in the middle of the test section $10 \mathrm{~cm}$ from the exit of the convergent nozzle. The paper sheets are dried in an oven and kept in a dessicator for at least forty-eight hours prior to performing the flame spread measurements.

The gas flow in the wind tunnel is supplied either from a centralized compressed air installation or from bottles of compressed oxygen and nitrogen. The gas flows of the individual gases are metered with calibrated critical nozzles and mixed at the settling chamber of the 


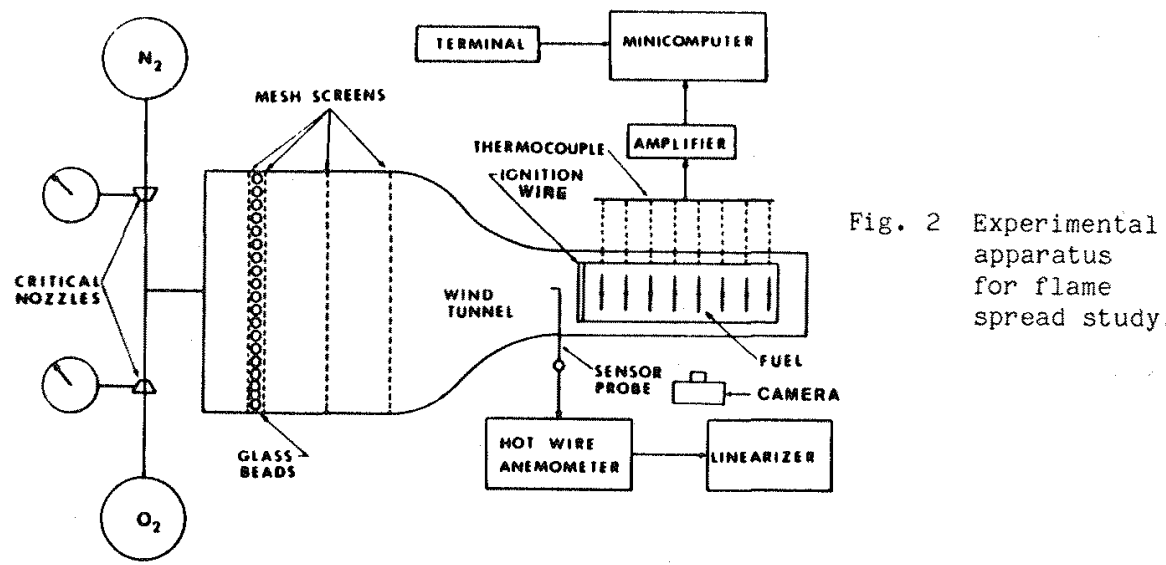

tunnel. Oxygen or nitrogen mixtures with concentrations accurate to within 1 percent are obtainable with the present installation. Maximum gas velocities obtainable in the present test section are of the order of 5 $\mathrm{m} / \mathrm{sec}$. The gas velocities are measured with a pitot tube instrument capable of measuring velocities down to $0.4 \mathrm{~m} / \mathrm{s}$, and $w i t h$ a hot wire anemometer. Prior to performing the experiments, extensive measurements were made of the velocity profiles along several planes of the test section to determine the characteristics of the flow. For the range of gas velocities used in these experiments, the flow showed a laminar character. The maximum flow Reynolds number at the downstream edge of the fuel specimen is of the order of $10^{3}$.

In the flow assisted mode of flame spread it is very important to have a uniform and well defined initiation of the flame spread process to assure two dimensionality $[2,9,10]$. In the present tests, the simultaneous ignition of the filter paper along its entire lower edge is achieved by means of an electrically heated nichrome wire. A thin layer of Duco-cement is applied to the paper where it touches the nichrome wire to favor the uniform ignition of the fuel. The cement burns very quickly and does not affect the flame spreading process. To initiate the spread of the flame the following steps are taken. With the fuel sheet positioned in the test section, the gas flow is established at the predetermined velocity and oxygen concentration. During the process of fuel ignition, the gas flow is bypassed to have a quiescent gas region near the leading edge of the fuel specimen and thus facilitates its ignition. On ignition, the heated nichrome wire pyrolyzes and ignites a thin region of the paper, initiating the spread process. As soon as ignition is observed, the bypass is closed and the data acquisition started.

The behavior of the flame is recorded with both direct photographs and thermocouple probing. In the thermocouple probing method, the rate of flame spread is measured from the temperature histories of thermocouples placed at fixed distances along the fuel surface [3]. Eight chromel-alumel thermocouples $0.0762 \mathrm{~mm}$ in diameter are placed in grooves made on the filter paper sheets each at distances $5.715 \mathrm{~cm}$ apart. The output from the thermocouples is amplified and processed in a real time data-acquisition system (PDP-11 mini-computer). The rate of spread of the pyrolysis front is obtained from the surface temperature histories by calculating the ratio of the distance between two consecutive thermocouples to the elapsed time 
of pyrolysis arrival to the thermocouples. The arrival of the pyrolysis front at the thermocouple position is characterized by the leveling of the temperature profile when the pyrolysis temperature of the fuel reaches an approximate constant value. The burnout location of the upstream fuel is recorded by observing the sudden decrease or increase in temperature due to the disappearance of the fuel or the contact of the trailing edge of the flame with the thermocouple, respectively. Motion pictures, taken at about $18 \mathrm{frames} / \mathrm{sec}$ frond of the fuel surface, provide another means for quantitative measurement of the flame spreading process. The motion pictures are used to evaluate the locations of the pyrolysis and burnt-out fronts, and the flame tip. Illumination of the paper surface with tungsten lamps, combined with the proper choice of exposure, yield well defined pyrolysis front locations. Photographically, this corresponds to the onset of the blackening of the paper. The burn-out location is more difficult to determine because in the burning region the paper breaks and curls, making it difficult to establish the real location of the fuel's disappearance. To overcome this problem, the location of the fuel burn-out is first assumed to coincide with the position of the initiation of the paper break out. The tests are then repeated up to five times to obtain an average of this distance. The distance obtained with this method just after the initiation of the paper breaking is subsequently matched with the burn-out distance obtained prior to the onset of the paper break out. The resultine correction is then applied to the rest of the burnmout data. The accurate determination of the flame tip position is also difficult because of the fluctuations of the flame tip. The results presented here are average values of distance obtained from color photographs of repeated tests.

\section{Results and Discussion}

The measurements of the distances of the pyrolysis front, $x_{p}$, the corresponding burn-out front, $x_{b}$, and flame tip, $x_{f}$, to the location of the flame spread initiation for flames spreading over the surface of filter paper sheets, are presented in Fig. 3 for several air flow velocities. The spread rates of each front can be deduced from these results by differentiating the corresponding distances with respect to time. The experiments are performed with the combustion tunnel in a vertical position to permit testing over the whole range of convective flow conditions (from free to forced). The pyrolysis front data is obtained using both the themocouple and photography methods. The data for the burn-out front and flame tip location are obtained primarily from the photographs, because this method seems to provide more reliable results. As explained above, because the method used to determine the locations of the burn-out front and the flame tip are not very accurate, the burn-out and flame tip data presented rere can only be considerea as approximate. The velocity of 2 $\mathrm{m} / \mathrm{s}$ is the maximum velooity for which the spread of the flame in air is observed. With thin fuels the length of the pyrolysis region remains relatively short due to the upstream consumption of the fuel, so the flame does not move into regions of larger boundary layer thickness. Consequently, extinction or non-flame propagation occurs at lower velocities than for the thick fuel sheets [6].

From the results presented in Fig. 3 it is seen that for air the flame spreading process is accelerative from ignition to approximately $15 \mathrm{~cm}$ downstream, becoming constant afterwards. This result follows the variation of the pyrolysis length $l_{p}=x_{p}-x_{b}$ and of the length of the flame over the not yet pyrolyzing fuel surface, thereafter called flame length $l_{f}=X_{f}-X_{p}$ with the distance from ignition. From Fig. 3 it is seen that 


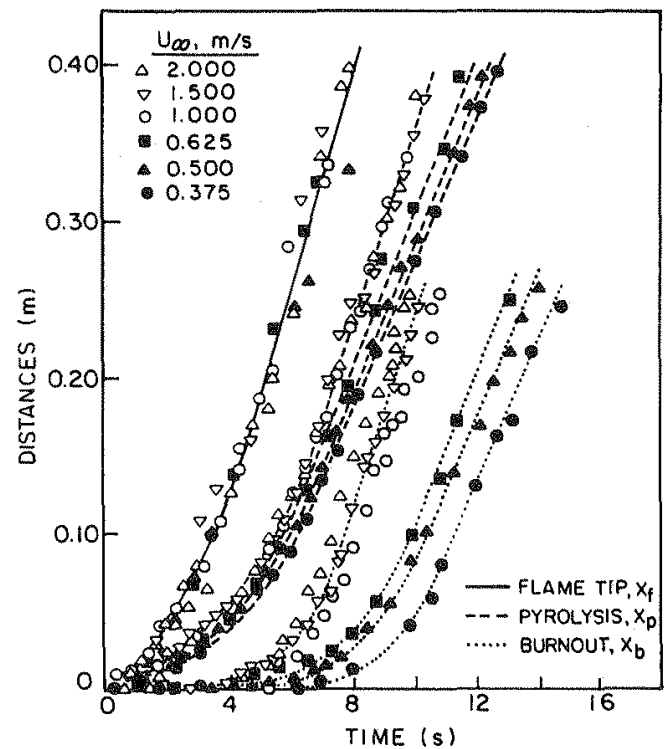

Fig. 3 Measurement of the variation with time of the pyrolysis flame and burn-out distances for flames spreading over thin paper sheets in a concurrent air flow.

both the pyrolysis and flame lengths increase rapidly during the initial period of the rlame spread process until burn-out of the fuel starts. After that, the rate of increase of these lengths decrease as the burn-out front progresses until finally they become practically constant at approximately $15 \mathrm{~cm}$ from ignition. The results of Fig. 3 also show that, for low flow velocity (mixed convection), the pyrolysis and flame lengths decrease as the flow velocity increases. Both lengths approximately become constant for forced flow conditions $\left(u_{\infty}>1 \mathrm{~m} / \mathrm{s}\right)$. The flame spread rate follows the variations of these lengths, increasing with the flow velocity for mixed flow conditions and becoming pratically constant for forced flow.

In Fig. 4, a logarithmic plot of the spread rate data for the initial accelerative period is presented. It is seen that there is an approximate power law dependence beween the pyrolysis distance and time of the form $x_{p}$ $\sim t^{n}$. The value of the exponent varies from 1.6 for natural convection to 2 for forced flow. The only theoretical models of the flow assisted mode of flame spread over thermally thin fuels that have been published to date are those of refs. [7 and 11] for natural convection and ref. [5] for forced convection. An overview of these models is given in ref. [12]. The analyses of refs. $[7,6]$ are very similar and both predict a fourth power law dependence between the pyrolysis distance and time. This is in disagreement with the results of $\mathrm{Fig}$. 4 for natural convection which gives a 1.6 power. On the otherhand, the analysis of ref. [5] for forced convection predicts a square power dependence for the pyrolysis distance with time which is in agreement with the results of Fig. 4. This last analysis, however, predicts a linear relationship between the pyrolysis distance and the free stream velocity. This prediction is in disagreement with the experimental results which show that the spread rate is practically independent of the flow velocity (Eig. 4). These comparisons indicate that the present theoretical models of the flow assisted spread of flames over thin fuels are not capable of predicting the process accurately and that some improved versions of these models are needed. 


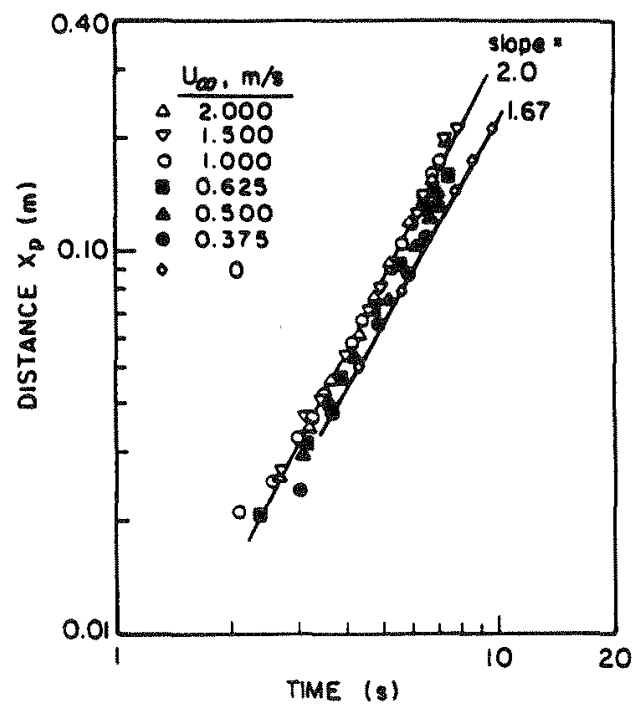

Fig. 4 Logarithmic plot of the accelerative flame spread data of $\mathrm{Fig} \cdot 3$.

Fig. 5

Dependence of the steady state flame spread rate on the concurrent flow velocity for various oxygen concentrations.

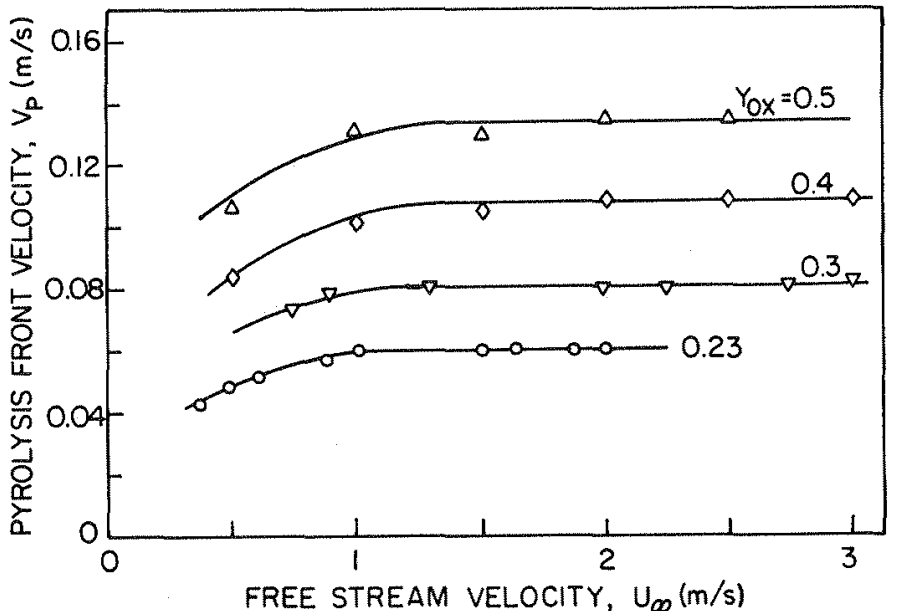

In Fig. 5 the rates of spread of the pyrolysis front, once the spread process has reached steady state, are presented as a function of the flow velocity for several oxygen concentrations. It is seen that for all oxygen concentrations the spread rate increases with the flow velocity for low flow velocities, and becomes practically independent of the gas velocity for forced flow conditions. Within the range of experimental conditions, the spread rate increases linearly with oxygen concentration.

In order to explain the nature of the above results, it is convenient to develop a simple model of the flame spread process over a thermally thin fuel. Assuming: that the primary controlling mechanism of flame spread is heat transfer from the flame to the non-burning material downstream from the pyrolysis front; that the heat flux from the flame is constant over the flame length and zero afterward; that the temperature of the fuel is uniform along its thickness (thermally thin); and that the combustible does 


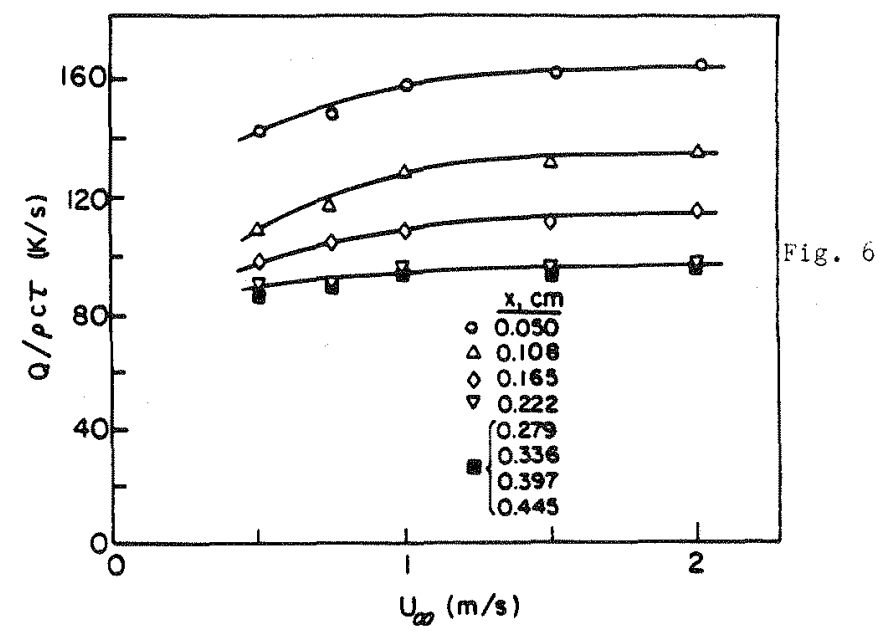

Measurements of the dependence of the local surface heat flux on the air flow velocity.

occurance of flame extinction the range of gas velocities tested (1m/s to 2 $\mathrm{m} / \mathrm{s}$ ) is very small. This, in conjunction with the fact that the experimental data has considerable scatter, and that the spread process may not behave as truly two-dimensional, suggests that the apparent independency of the heat flux with the flow velocity may not be totally true and that indeed the heat flux is dependent on the flow velocity as expected from the boundary layer analysis predictions.

The experimental results for the flame spread rate can be explained phenomenologically with the help of Eq.(1) (or Eq.(2)) and the results of Figs. 3 and 6 . The initial accelerative period of the flame spread rate (Figs. 3 and 4 ) is due to the increase of the pyrolysis length which in turn results in the increase of the flame length (Fig. 3) and consquently of the total heat flux at the fuel surface. The slight decrease of the local heat flux with the distance from ignition (Fig. 6) is counteracted by the increase of the flame length. Once the fuel starts to be depleted in the upstream region, the rate of increase of the pyrolysis length decreases and so does the spread rate. As the rate of spread of the burn-out front approaches that of the pyrolysis front, the pyrolysis length becomes constant. This results in a steady state flame spread process. Similarly, the increase of the spread rate with $u_{\infty}$ for low flow velocities is due to the increase of the surface heat flux (Fig. 6) which counteracts the slight decrease of the flame length (Eig.3). For larger flow velocities $\left[u_{\infty}>1\right.$ $m(s)]$, the pyrolysis and flame length and the surface heat flux become approximately constant, and consequently so does the rate of flame spread.

With regard to the independence of the flame spread rate on the gas velocity for forced flow conditions, it should be pointed out that the reasons given above for this result, i.e: the constancy of $l_{p}$, $l_{f}$ and $q{ }_{f}$, may not be totally accurate. As explained before, the present measurements have some scatter due to experimental difficulties and the variation of the above parameters with $u_{\infty}$ is expected to be small due to the small range of velocities tested. Therefore, there is the possibility that the above parameters are not truly constant but vary with length and velocity according to the boundary layer predictions. Under these conditions the dependence of the flame spread rate on the flow velocity would be the result of the following mechanism. As the flow velocity increases the 
not vaporize until its temperature reaches a given value; and that the spread process is two dimensional, an energy balance for a control volume in the solid downstream from the pyrolysis front (Fig. 1) gives

$$
\rho \circ \tau V_{p}\left(T_{V}-T_{\infty}\right)=\dot{q}_{f} l_{f}
$$

In the above equation, $\rho, c, \tau, T$ and $T_{\infty}$ are respectively the density, specific heat, thickness, pyrolysis and initial temperature of the fuel; $V_{p}$ is the spread rate of the pyrolysis front; q"f the heat transfer from the flame to the fuel surface by radiation and convection; $l_{f}$ is the flame length over the not yet pyrolizing surface. The spread rate can also be expressed in terms of the pyrolysis length by replacing $d_{f}$ in Eq. (1) by the relation $l_{f}=C l_{p}^{n}$ with $n \leqq 1,[2,11,12],(n \approx 1.1$ from the results of Fig.3).

The heat flux at the surface, neglecting radiation, can be expressed in the form $\dot{q}^{\prime \prime}{ }^{\sim} \lambda_{g}\left(T_{f^{-}} T_{v}\right) / \delta$, where $\lambda_{g}$ is the thermal conductivity, $\mathrm{T}_{\mathrm{f}}$ is the flame temperature and $\delta$ is the flame standmof distance. Substituting this relation in Eq. (1), the following expressions is obtained for the rate of spread of the pyrolysis front,

$$
v_{p}-\frac{\lambda_{g}\left(T_{f}-T_{v}\right)}{\operatorname{por}\left(T_{V}-T^{\infty}\right)}\left(l_{f} / \delta\right)
$$

The variation of $v_{p}$ with the pyrolysisfront distance (or time) and with the flow velocity will depend on the respective variations of $l_{f}$ and $\delta$. The variation of the former parameter can be deduced from the results of Fig. 3 and of the latter from the results of Fig. 6 .

In Fig. 6, the surface heat flux calculated from surface temperature histories at different locations along the fuel surface is plotted as a function of the free stream velocity. It is seen that the heat flux decreases initially with the distance from the fuel ignition location and becomes approximately constant after a distance of approximately $15 \mathrm{~cm}$. This trend is in qualitative agreement with the predictions of boundary layer analyses of burning surfaces where a scaling law for of the form $\delta$ $\sim l_{p}{ }^{a} u_{\infty} b$ can be deduced [12]. The initial increase of the pyrolysis length (Fig.3) results in an increase of the flame stand-of $f$ distance and, consequently, in a decrease of heat flux. Once lp becomes constant, so does o and consequently $\dot{q}^{\prime \prime}$. From the dejendence on $u_{\infty}$, it is seen that the heat flux increases with the velocity for low velocities, but becomes practically constant for $u_{\infty}>1 \mathrm{~m} / \mathrm{s}$. This last result shows agreement of the above dependence of $\delta$ on $l_{p}$ but seems to disagree with the predicted dependence on $u_{\infty}$. From the results of Fig. 3 it is seen that, for low velocities, as $u_{\infty}$ increases $h_{p}$ decreases, which results in a decrease of the flame stand-of $\mathrm{f}$ istance and consequently in an increase of the heat flux. For large flow velocities, however, the pyrolysis length is practically independent of the flow velocity. Since the heat flux is also very weakly dependent on $u_{\infty}$, it appears that the heat flux is primarliy dependent on $b$ and not on $u_{\infty}$. With regard to the apparent weak dependence of the heat flux on the flow velocity, it should be mentioned that the observed strong variation of the flame spread data with the flow velocity occurs during the transition from natural to forced convection, where markedly different flow patterns are expected. However, under forced flow conditions the variation of the flame spread parameters with the flow velocity is not very strong ( $\sim 1 / 2$ power). Futhermore, because of the 
thickness of the boundary layer and consequently the flame stand-off distance decrease, which results in an increase of the heat transferred from the flame to the fuel. This increase in heat transfer has a dual effect. While it increases the heat flux at the non-burning fuel surface, it also increases the gasification rate of the burning surface. A larger mass burning rate causes an increase in the rate of spread of the burn-out front that tends to decrease the length of the pyrolysis region and conseqently of the flame length. As it is seen from Eq. (1) both effects - the increase of the heat flux and the decrease of the pyrolysis length (or flame length) - counteract each other, and, depending on their relative variation, the spread rate would either increase, remain constant, or even decrease. The results of Figs. 3 and 5 seem to indicate that at large velocities (forced flow) both effects would balance each other.

A correlation, using Eq. (2) of the flame spread rate data of Fig. 5 with $\ell_{f} / \delta$ assumed constant for forced convection, is presented in Eig. 7 . The properties used in the computation of the correlation are $\lambda_{\mathrm{g}}=0.046$ $\mathrm{J} / \mathrm{m}-\mathrm{sec} \mathrm{K}, \mathrm{c}_{\mathrm{p}}=1.06 \mathrm{~kJ} / \mathrm{kg} \mathrm{K}, \rho \tau=18.5 \mathrm{k} 10^{-3} \mathrm{~g} / \mathrm{cm}^{2}$. The flame temperature $T_{f}^{p}$ is calculated with the equation [15]

$$
T_{f}=T_{V}+\frac{\left(T_{\infty}-T_{V}\right)+\left(Y_{O \infty} s / c_{p}\right)\left(\Delta H_{c}-L\right)}{1+Y_{O \infty} s}
$$

this corresponds to the adiabatic flame temperature for constant specific heat of the products of combustion. The data used in calculating the flame temperature are: $s=0.844, \Delta \mathrm{H}_{\mathrm{c}}=1.674 \times 10^{4} \mathrm{KJ} / \mathrm{kg}$ fuel, $\mathrm{L}=753 \mathrm{KJ} / \mathrm{kg}$, $T_{\infty}=298^{\circ}$ and $T_{V}=618^{\circ} \mathrm{K}$. From the results of $\mathrm{fig} .7$ it is seen that except at low flow velocities $\left(u_{\infty}<1 \mathrm{~m} / \mathrm{s}\right)$, where buoyancy effects become important, Eq. (2) correlates very closely the flame spread data of Fig. 5 . Since Eq. (2) does not include radiation heat transfer from flame to fuel, the good'correlation of the experimental data indicates that radiation is not an important mechanism of heat transfer for the present experiments. This is probabiy due to the small scale of the experimental set up, and to the fact that the fuel burn out limits the size of the flame. It is interesting to note that the analysis of ref. [14] for the spread of a flame over a thin fuel in an opposed flow predicts a spread rate that is

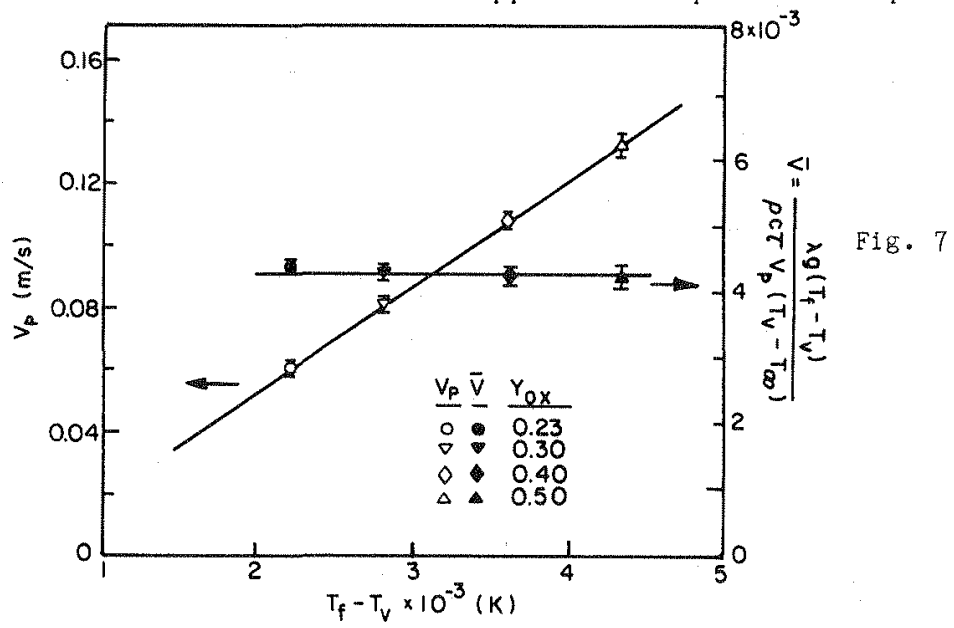

Correlation of the rate of spread of the pyrolysis front data with the model prediction. 
also given by an equation as in Eq. (3) and that is independent of the flow velocity. As it is explained in ref. [6] this is understandable. A lump energy balance of the gas phase for the opposed and concurrent modes of spread show that while the convective terms have opposite signs, the temperatures also have opposite signs, and both cancel each other. The result is a formula that is applicable to both models of heat transfer.

Conclusion

The measurement of the rates of flame spread over the surface of thin filter paper sheets and their comparison with currently available theoretical models show deficiencies in the predictive capabilities of the models. It appears that the major problem comes from the prediction of the variation with time and of the dependence on the flow velocities of the length of the pyrolysis region. This length determines in its turn the length of the flame and consequently the overali heat flux on the fuel surface. Thus an accurate prediction of the pyrolysis and flame lengths is imperative to preaict accurately the rates of flame spread.

\section{Acknowledgements}

The authors gratefully acknowledge the comments and suggestions of Professors T. Hirano, K. Sato, and P.J. Pagni concerning the experimental procedure and results. The research was supported by the National Bureau of Standards under grant number: NB83NADA 4020.

\section{References}

1. Hansen, A. and Sibulkin, M.: Combustion Science and Technology, 9 , p. $173(1974)$.

2. Orlof', L., de Ris, J.N. and Markstein, G.H.: Fifteenth Symposium on Combustion, p. 183; The Combustion Institute (1975).

3. Fernandez-Pello, A:C.: Combustion Science and Technology, 17, p. $87(1977)$.

4. Alpert, R.L.: ASME-AICHE National Heat Transfer Conference, ASME publication no. 79-MT-28 (1979).

5. Fernandez-Pel10, A.C.: Combustion and Flame, 36, p. 63 (1979).

6. Loh, H.T. and Fernandez-Pel10, A.C.:"A Study of the Controlling Mechanisms of Flow Assisted Flame Spread," The Twentieth (International) Symposium on Combustion, The Combustion Institute, (in press) (1984).

7. Markstein, G.H. and de Ris, J.H.: (a) Fourteenth Symposium (International) on Combustion, p. 1085. The Combustion Institute (1973); (b) also FMRC Report 20588 (1972).

8. Hirano, T. and Sato, Ko: Fifteenth symposium (International) on Combustion, p. 233, The Combustion Institution (1975).

9. Hirano, T., Noreikis, S.E. and Waterman, T.E.:Combustion and Flame, 22 , p. $353(1974)$.

10. Fernandez-Pello, A.C. and Hirano,T.:Combustion Science and Technology, 32 , p. 1 (1983).

11. Fernandez-Pello, A.C.: Combustion and Elame, 31, p. 135 (1978).

12. Fernandez-Pello, A.C.: Combustion, Science and Technology, 39 , p. $119(1984)$.

13. Pagni, P.J.:Fire Safety Journal, 3, 2-4, $273(1981)$.

14. de Ris, J.N.:Twelfth Symposium on Combustion, p. 241, The Combustion Institute (1969).

15. Altenkirch, R.A., Eichnorn, R. and Shang, P.C.: Combustion and Flame, 37 , p: 71 (1980). 